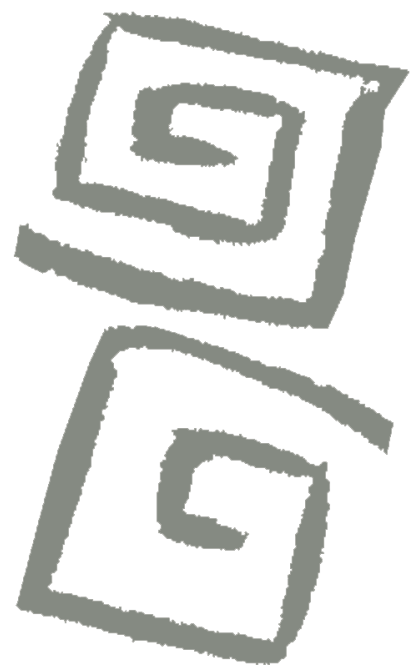

\title{
Cambios en la tendencia de mortalidad por suicidio en Chile, 1997-2018
}

\section{Changes in suicide mortality trends in Chile, 1997-2018}

Carolina Vidal' ${ }^{1}$, Carlos Faunes², Carol Toro Huerta ${ }^{3}$, Cristóbal Ruiz-Tagle ${ }^{4}$, Lorena Hoffmeister ${ }^{5}$

${ }^{\top}$ Magíster en Salud Pública y Planificación Sanitaria. Investigador-docente, Escuela de Salud Pública, Universidad Mayor Santiago, Chile. $\bowtie$ iD

${ }^{2}$ Magíster en Salud Pública y Planificación Sanitaria, Escuela de Salud Pública, Universidad Mayor Santiago, Chile. $\triangle$

${ }^{3}$ Autora de correspondencia. Magíster en Políticas Públicas. Docente, Escuela de Obstetricia y Puericultura, Facultad de Ciencias, Universidad Mayor Santiago, Chile. $\bowtie$ iD

${ }^{4}$ Magíster en Salud Pública y Planificación Sanitaria. Investigador-docente, Escuela de Salud Pública, Universidad Mayor Santiago, Chile. $\triangle$ iD

${ }^{5}$ Doctora en Biomedicina. Directora, Escuela de Salud Pública, Universidad Mayor, Santiago, Chile. $\bowtie$ iD
RESUMEN Con base en el registro de defunciones del Departamento de Estadísticas e Información del Ministerio de Salud de Chile, se realizó un estudio ecológico de series de tiempo para determinar cambios en la tendencia de las tasas de mortalidad por suicidio en Chile según sexo y edad, en el periodo 1997-2018. Los resultados muestran que la tasa de mortalidad en hombres para el año 2018 fue de 20,1 por 100.000, siendo casi 5 veces más que la de mujeres. La tendencia en ambos sexos muestra un descenso en el porcentaje de cambio anual del $-5,4 \%$ [IC95\% $(-12,9 ; 1,9)]$ en el periodo 2009-2013. Los hombres, en el mismo periodo, muestran un porcentaje de cambio anual del $-5,8 \%$ [IC95\% $(-12,5 ; 2,3)]$, mientras que en las mujeres, para el periodo 2008- 2018, es $-4,0 \%$ [IC95\% $(-5,8 ;-2,2)]$. No se observaron cambios en la tendencia en los hombres de 60 o más años, siendo este grupo el que presenta las tasas más altas. Si bien esta disminución se registra con posterioridad a la implementación de políticas enfocadas en factores de riesgo de suicidio, es necesario evaluar sistemáticamente dichas políticas e implementar otras con enfoque en las poblaciones identificadas con mayor riesgo.

PALABRAS CLAVES Suicidio; Tasa de Mortalidad; Chile.

ABSTRACT Using information from the mortality database at Chile's Department of Statistics and Health Information (Ministry of Health), an ecological time-series study was conducted to determine changing trends in suicide rates by sex and age group in Chile from 1997 to 2018. Results show that the mortality rate for men in 2018 was 20.1 per 100,000, almost five times higher than the rate for women. Trends in both sexes show a decrease in aver-age annual percent change of $-5.4 \%$ [CI95\% $(-12.9 ; 1.9)]$ between 2009 and 2013. Over the same period, the average annual percent change for men was $-5.8 \%$ [Cl95\% $(-12.5 ; 2.3)]$, while for women it was -4.0 [CI95\% (-5.8; -2.2)] between 2008 and 2018. No changes have been observed in trends for men aged 60 and over, the group with the high-est rates. Although suicide rates declined following the implementation of policies focus-ing on risk factors for suicide, it is necessary to evaluate the implementation of these pol-icies and devise similar actions geared toward populations with greater risk of suicide.

KEY WORDS Suicide; Mortality Rate; Chile. 


\section{INTRODUCCIÓN}

El suicidio es reconocido como un problema crítico de salud pública por la Organización Mundial de la Salud (OMS), en su plan de acción integral de salud mental ${ }^{(1)}$. El suicidio se ha definido como una muerte causada por una lesión autoinfligida intencional ${ }^{(2)}$. A nivel mundial, el suicidio representa el 1,4\% de las muertes ${ }^{(3)}$, y se encuentra entre las 20 principales causas de mortalidad ${ }^{(4)}$. El número total de muertes por suicidio aumentó globalmente entre 1990 y 2016 en un 6,7\%. Estimaciones realizadas mediante estadística bayesiana muestran una mayor tasa para los hombres con 15,6 muertes por 100.000 (intervalo de incertidumbre del 95\% [13,7; $17,2]$ ) que para las mujeres con 7,0 (intervalos de incertidumbre del $95 \%[6,5 ; 7,4])$, además la disminución en la tasa desde 1990 a 2016 fue menor en los hombres $23,8 \%$ que para mujeres $49,0 \%{ }^{(5)}$.

La mortalidad por suicidio también varía sistemáticamente por región. América Latina ha presentado tasas de mortalidad por suicidio menores que el resto del mundo(4); sin embargo, los datos de mortalidad han sido descritos como irregulares, especialmente al compararlos con los datos de los países europeos $^{(6)}$. En la región, también existen marcadas disparidades en las tasas de suicidio entre los distintos países, incluso entre algunos con niveles similares de desarrollo ${ }^{(7)}$. Para el caso de Chile, entre las causas de muerte no natural el suicidio es la segunda causa de muerte a nivel país, superada solo por aquellas muertes producto de accidentes del tránsito ${ }^{(8)}$. Según el informe de la Organización para la Cooperación y el Desarrollo Económicos (OCDE), en los países miembros, desde 1990 las tasas de suicidio han decrecido en promedio en un $20 \%$. Sin embargo, contrario a esta tendencia, en Chile las tasas han continuado en aumento, alcanzando un crecimiento del $90 \%$ entre los años 1990 y $2011^{(9)}$. Los factores individuales y ambientales que contribuyen al suicidio varían sustancialmente entre países y regiones ${ }^{(10)}$. La evidencia muestra diferencias de forma sistemática según edad, sexo y medio de suicidio(11). En los países de altos ingresos, los hombres de mediana edad y adultos mayores han presentado las tasas más altas de suicidio. Sin embargo, las tasas de suicidio juvenil son una causa creciente de preocupación, siendo el suicidio la segunda causa de muerte en individuos de 15 a 29 años. La razón de suicidios entre hombres y mujeres ha sido más alta en países de ingresos altos versus bajos y medianos ${ }^{(1)}$.

Acciones propuestas por los países miembros de la OMS han incluido el desarrollo e implementación de estrategias nacionales de prevención del suicidio, con un enfoque en las poblaciones identificadas con mayor riesgo de suicidio. Las estrategias nacionales de prevención del suicidio son esenciales para lograr el objetivo final de la reducción del suicidio. Los países miembros de la OMS se han comprometido en el Plan de acción de salud mental 2013-2020 para trabajar hacia el objetivo mundial de reducir la tasa de suicidios en los países en un 10\% para $2020^{(12)}$

En Chile, actualmente no existe una política pública integral de salud mental; sin embargo, se han implementado diversas políticas que abordan el suicidio o la prevención de sus causas. En el año 2001, se incorporó el Programa Nacional de Detección, Diagnóstico y Tratamiento de la Depresión a la atención primaria de salud; en el año 2006, se incorporó a las Garantías Explicitas en Salud de Chile la depresión para personas mayores de 15 años, posteriormente se incluyó esquizofrenia, trastorno bipolar en mayores de 15 años y consumo problemático de drogas en menores de 20 años $^{(13)}$. A partir del año 2013, Chile ha diseñado un Programa Nacional de Prevención del Suicidio, el cual se enmarca en el objetivo sanitario de fomentar las competencias en la población para el autocuidado y la protección de estilos de vida y entorno saludables ${ }^{(14)}$. Dado los esfuerzos en la prevención del suicidio y de sus factores de riesgo, el objetivo de este estudio fue determinar cambios en la tendencia de las tasas de mortalidad por suicidio en Chile según sexo, durante el periodo 1997-2018. 


\section{MATERIAL Y MÉTODOS}

Se realizó un estudio ecológico, con base en la información de mortalidad por suicidio entre los años 1997-2018, extraída de los registros de defunciones del departamento de Estadísticas e Información de Salud del Ministerio de Salud de Chile ${ }^{(15)}$. Los registros de defunciones incluyen la causa básica de defunción, codificada a partir de la Clasificación Internacional de Enfermedades en su décima versión $(\mathrm{CIE}-10)^{(16)}$. El registro de defunciones es rutinario y obligatorio para todas las muertes acontecidas en territorio nacional. De estos registros se seleccionaron los casos de 15 o más años, con causa externas de mortalidad asociada a los códigos X60-X84 de la CIE-10, definidos como lesiones autoinfligidas intencionalmente. En Chile, la cobertura del registro de las causas de mortalidad se considera de alta calidad, y se registran más del $98 \%{ }^{(17)}$. Se revisó la cantidad de registros con clasificación de eventos con intención indeterminada (códigos Y10-Y34 de la CIE10). Se encontró un total de 8.233 registros de estas causas, de las cuales 8.123 ocurren entre 1997 y 2001, mientras que las restantes ocurrieron en 2004. No existieron más registros de estas causas durante el periodo de estudio. Esta disminución tan drástica fue atribuida, en gran medida, a los cambios en el sistema de validación de información, tales como el mejoramiento en el análisis de los datos contenidos en el certificado de defunción y el análisis simultáneo de las bases de datos de defunciones con otras fuentes de información ${ }^{(18)}$.

La población bajo riesgo se obtuvo de las proyecciones poblacionales basadas en el último censo del año 2017, publicado por el Instituto Nacional de Estadística de Chile ${ }^{(19)}$. Con estos datos se calcularon las tasas brutas y específicas por sexo y grupos de edad. Para calcular las tasas ajustadas por edad se utilizó como población de referencia la estimada por Naciones Unidas en el año $2018^{(20)}$.

La tendencia temporal de la tasa de mortalidad se analizó mediante la regresión de Poisson, que describe diferentes patrones de evolución temporal de datos de mortalidad $^{(21)}$. Para identificar los períodos entre 1997-2018 en que se produjeron cambios significativos en la tasa de mortalidad, se construyó un modelo de regresión joinpoint. Este modelo, también Ilamado "modelo segmentado de Poisson", identifica el momento en que se producen cambios significativos en la tendencia y, además, estima la tendencia observada en dicho intervalo. Es uno de los métodos más empleados para estimar los cambios en la tendencia de tasas de mortalidad e incidencia, ya que consigue un mejor ajuste comparado con modelos lineales, que reducen la tendencia a una sola regresión $^{(20,21)}$. Para la selección del número de joinpoint se utilizó el método de estimación de grid search. Como medida de resumen de los modelos de regresión joinpoint se han utilizado los porcentajes de cambio anual (PCA), tanto de la tasa global de mortalidad por suicidio como de las tasas específicas por sexo y grupo de edad, se definió un nivel de significación estadística de 0,05. Para el análisis se utilizó el programa de análisis de tendencias Joinpoint versión 4.8.0.1 $1^{(22)}$.

Para este estudio se utilizaron registros de datos de uso público, obtenidos del Ministerio de Salud de Chile, por lo que los datos fueron confidenciales y anónimos según lo dispuesto en la Ley $N^{\circ} 17$ 374, Artículo 29 ${ }^{(23)}$.

\section{RESULTADOS}

Durante el periodo de estudio hubo 37.343 muertes por suicidio en Chile en personas de 15 o más años, 30.906 en hombres y 6.437 en mujeres. La Figura 1, muestra los cambios en la tendencia de la tasa de mortalidad por suicidio en ambos sexos. En el año 1997, la tasa de mortalidad por suicidio fue de 8,54 por 100.000 habitantes mientras que, en 2018, fue de 11,91 por 100.000 habitantes. Se observaron tres cambios (joinpoint) en la tendencia. El primer segmento (periodo) es 1997-2001, en el cual se observó un aumento en la tendencia temporal con un PCA de 12,4 (IC95\% [5,4; 19,9] valor de 


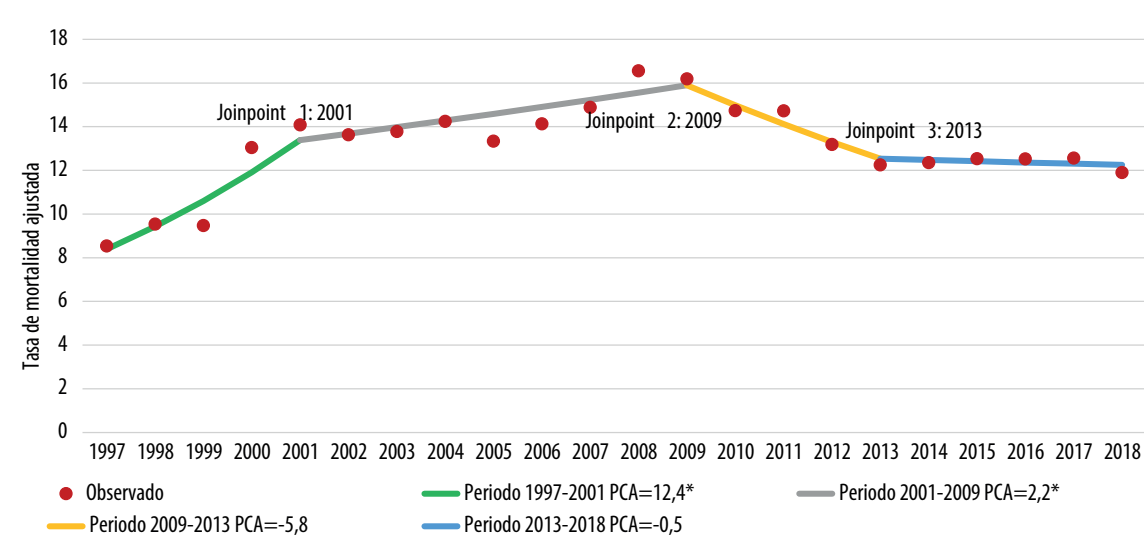

Figura 1. Evolución de la tasa de mortalidad de suicidio en ambos sexos. Chile, 1997-2018.

Fuente: Elaboración propia con base en los registros de defunciones del departamento de Estadísticas e Información de Salud del Ministerio de Salud de Chile(15).

*Indica que el porcentaje de cambio anual (PCA), significativamente distinto de cero, considerando un nivel de significación estadística de 0,05.

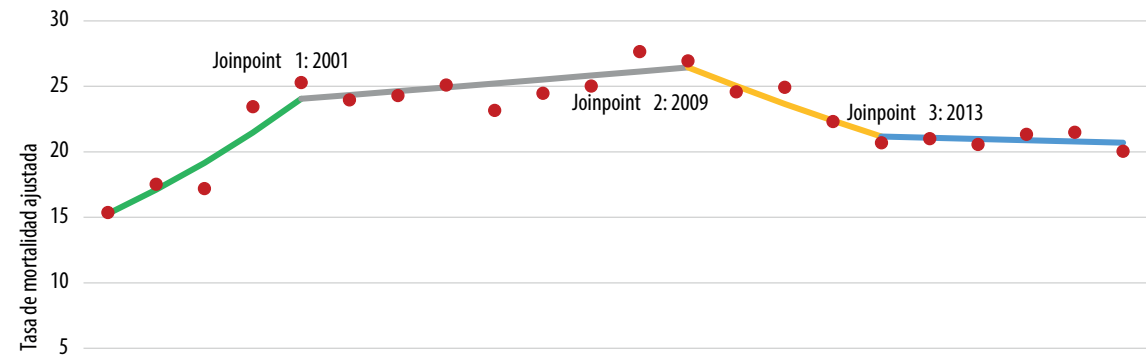

01997199819992000200120022003200420052006200720082009201020112012201320142015201620172018

- Observado

Periodo 1997-2001 PCA=12,0

Periodo 2001-2009 PCA=1,2*

Periodo 2009-2013 PCA=-5,4 Periodo 2013-2018 $\mathrm{PCA}=-0,5$

Figura 2. Evolución de la tasa de mortalidad por suicidio especifica en hombres, Chile 1997-2018.

Fuente: Elaboración propia con base en los registros de defunciones del departamento de Estadísticas e Información de Salud del Ministerio de Salud de Chile(15).

*Indica que el porcentaje de cambio anual (PCA), significativamente distinto de cero, considerando un nivel de significación estadística de 0,05.

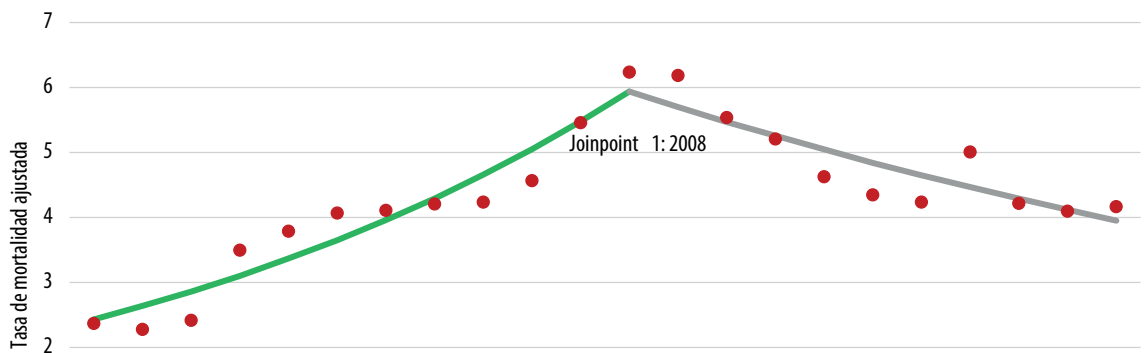

1

1997199819992000200120022003200420052006200720082009201020112012201320142015201620172018 - Observado Periodo 1997-2008 PCA=8,5* Periodo 2008-2018 PCA=-4,0*

Figura 3. Evolución de la tasa de mortalidad por suicidio especifica en mujeres, Chile 1997-2018.

Fuente: Elaboración propia con base en los registros de defunciones del departamento de Estadísticas e Información de Salud del Ministerio de Salud de Chile ${ }^{(15)}$.

*Indica que el porcentaje de cambio anual (PCA), significativamente distinto de cero, considerando un nivel de significación estadística de 0,05. 
$p<0,001$ ), el siguiente periodo 2001-2009, mostró otro un aumento con un PCA de 2,2 (IC95\% $[0,0 ; 4,4]$ valor de $p<0,001)$. En el tercer segmento, 2009-2013, hubo un descenso en la tasa, con un PCA de 5,8 (IC95\% [-12,9; $1,9]$ valor de $p>0,05)$. Finalmente, en el último periodo 2013-2018, se observó un PCA cercano a cero. Solo los cambios observados en el primer y segundo segmento fueron estadísticamente significativos (valor de $p<0,05$ ).

La Figura 2 y la Figura 3 muestran la tendencia de mortalidad por suicidio según sexo. Los hombres presentaron tres joinpoint ubicados en los mismos años que en el análisis general. El primer periodo, 1997-2001, mostró un PCA de 12,0 (IC95\% [5,1; 19,3] valor de $p<0,001)$, siendo este el único estadísticamente significativo, mientras que en el tercer segmento 2009-2013, si bien presentó un descenso, ha sido más desacelerado con un PCA de $-5,4$ (IC95\% $[-12,5 ; 2,3]$ valor de $p>0,05)$. Las mujeres solo presentaron un joinpoint en el año 2008. El primer periodo 1997-2008 mostró un aumento sostenido, con un PCA de 8,5 (IC95\% $[6,2 ; 10,8]$ valor de $p<0,001)$ y el segundo periodo 20082018, una disminución con un PCA de $-4,0$ (IC95\% $[-5,8 ;-2,2]$ valor de $p<0,001)$.

La Figura 4 y la Figura 5 muestran la evolución de las tasas de mortalidad específicas

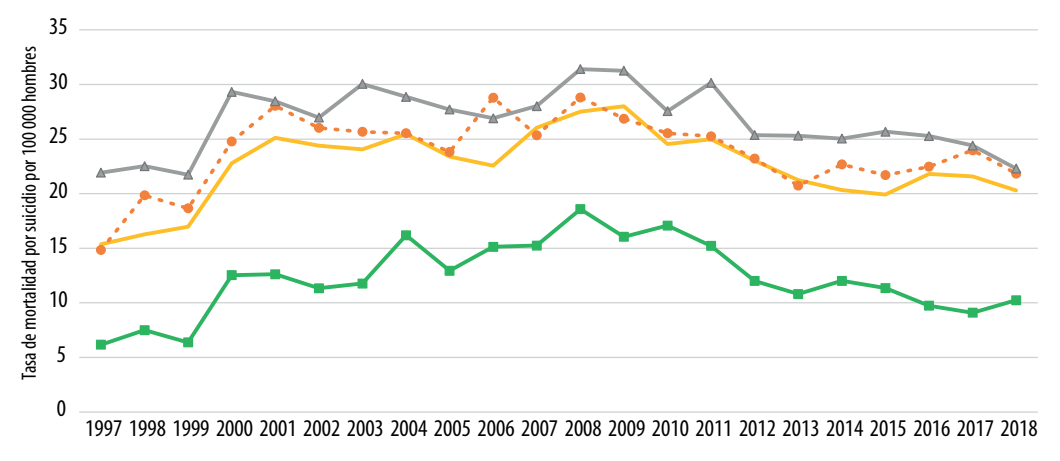

$\longrightarrow$-15-19 años $\longrightarrow$ 20-39 años $\quad \cdots$ 40-59 años $\longrightarrow 60$ o más años

Figura 4. Evolución de la tasa de mortalidad especifica en hombres por grupo de edad, Chile 1997-2018. Fuente: Elaboración propia con base en los registros de defunciones del departamento de Estadísticas e Información de Salud del Ministerio de Salud de Chile ${ }^{(15)}$.

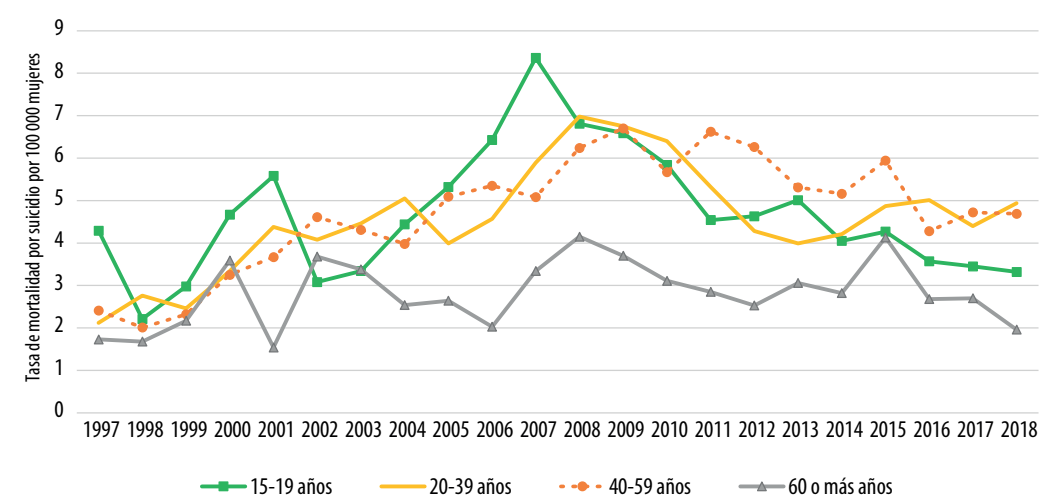

Figura 5. Evolución de la tasa de mortalidad especifica en mujeres por grupo de edad, Chile 1997-2018.

Fuente: Elaboración propia con base en los registros de defunciones del departamento de Estadísticas e Información de Salud del Ministerio de Salud de Chile ${ }^{(15)}$. 
Tabla 1. Número de joinpoint y porcentaje de cambio anual por grupo de edad, según sexo. Chile, 1997-2018.

\begin{tabular}{|c|c|c|c|c|c|c|}
\hline Sexo & Grupo de edad & $\begin{array}{c}\mathrm{N}^{\circ} \mathrm{de} \\
\text { joinpoint }\end{array}$ & Periodo & PCA & IC $95 \%$ & valor de $p$ \\
\hline \multirow{11}{*}{ Hombres } & \multirow{2}{*}{15 a 19 años } & \multirow{2}{*}{1} & $1997-2008$ & 7,9 & 11,$3 ; 5,2$ & $<0,001$ \\
\hline & & & $2008-2018$ & $-6,9$ & $-9,8 ;-3,8$ & $<0,001$ \\
\hline & \multirow{5}{*}{20 a 39 años } & \multirow{5}{*}{4} & $1997-2001$ & 14,5 & 7,$0 ; 22,5$ & 0,001 \\
\hline & & & $2001-2006$ & $-0,9$ & $-6,6 ; 5,0$ & 0,718 \\
\hline & & & $2006-2009$ & 5,6 & $-11,5 ; 0,7$ & 0,490 \\
\hline & & & 2009-2014 & $-6,2$ & $-11,5 ;-0,7$ & 0,032 \\
\hline & & & 2014-2018 & 1,2 & $-4,7 ; 7,3$ & 0,665 \\
\hline & \multirow{2}{*}{ 40-59 años } & \multirow{2}{*}{1} & 1997-2001 & 15,7 & 5,$3 ; 27,0$ & 0,004 \\
\hline & & & $2001-2018$ & $-1,3$ & $-2,1 ;-0,5$ & 0,004 \\
\hline & \multirow{2}{*}{60 o más } & \multirow{2}{*}{1} & 1997-2001 & 2,3 & 0,$6 ; 4,0$ & 0,009 \\
\hline & & & 2001-2017 & $-2,8$ & $-4,3 ;-1,3$ & $<0,001$ \\
\hline \multirow{8}{*}{ Mujeres } & \multirow{2}{*}{15 a 19 años } & \multirow{2}{*}{1} & 1997-2007 & 8,6 & 4,$0 ; 13,5$ & $<0,001$ \\
\hline & & & 2007-2018 & $-6,8$ & $-10,1 ;-3,3$ & $<0,001$ \\
\hline & \multirow{3}{*}{ 20-39 años } & \multirow{3}{*}{2} & $1997-2009$ & 8,9 & 6,$4 ; 11,5$ & $<0,001$ \\
\hline & & & 2009-2013 & $-12,6$ & $-25,8 ; 3,1$ & 0,100 \\
\hline & & & 2013-2018 & 4,2 & $-3,4 ; 12,5$ & 0,263 \\
\hline & \multirow{2}{*}{ 40-59 años } & \multirow{2}{*}{1} & 1997-2009 & 8,8 & 6,$2 ; 11,4$ & $<0,001$ \\
\hline & & & 2009-2018 & $-4,2$ & $-6,7 ;-1,6$ & 0,003 \\
\hline & 60 o más años & 0 & $1997-2018$ & 0,5 & $-1,4 ; 2,5$ & 0,586 \\
\hline
\end{tabular}

Fuente: Elaboración propia con base en los registros de defunciones del departamento de Estadísticas e Información de Salud del Ministerio de Salud de Chile ${ }^{(15)}$.

PCA = Porcentaje de cambio anual. IC 95\%= Intervalo de confianza del 95\%.

por edad según sexo. Además, la Tabla 1 muestra el número de joinpoint estimados para cada grupo de edad según sexo. En hombres, el grupo de edad que presentó más cambios (joinpoint) en su tendencia fue el de 20 a 39 años, lo mismo puede observarse en mujeres. Para los hombres pertenecientes al grupo etario de 60 y más años, se observó un descenso durante el último periodo con un PCA de -2,8 (IC95\% [-4,3; -1,3] valor de $p<0,001)$, mientras que la tendencia para las mujeres del mismo grupo ha permanecido constante. Cabe señalar que, en todos los grupos etarios, las tasas son superiores en hombres, y se observa que esta brecha se presentó con mayor magnitud en los hombres de 60 y más años.

\section{DISCUSIÓN}

Este estudio evaluó los cambios de la tendencia en las tasas de mortalidad por suicidio en Chile según sexo y edad durante los años 1997-2018. El PCA para todo el periodo fue de $-0,33$. Se observaron cuatro quiebres en la tendencia durante el periodo estudiado, de los cuales dos fueron estadísticamente significativos. En ambos periodos, aumentó la tasa de mortalidad, con un PCA de 12,4 para el periodo 1997-2001 y un PCA de 2,2 para el periodo 2001-2009. En los últimos nueve años, se observó un leve descenso de la tasa de mortalidad ajustada; sin embargo, este no fue estadísticamente significativo. Según 
información reportada por la OCDE, entre los años 1990 y 2013, las tasas de suicidio disminuyeron en 27 de los 36 países miembros; sin embargo, Chile registró la segunda alza más importante del periodo después de Corea del Sur ${ }^{(9)}$. Esta diferencia respecto de nuestro estudio radicaría en las metodologías utilizadas para analizar las tendencias. La OCDE consideró el periodo 1990-2013 como un continuo y el presente análisis evaluó las tendencias para cada cambio ocurrido en el periodo.

La evidencia señala que existen diferencias en las conductas suicidas entre hombres y mujeres, si bien las mujeres presentan con mayor frecuencia ideaciones e intentos suicidas respecto de los hombres, estos últimos resultan ser más efectivos debido a la mayor letalidad de los métodos utilizados ${ }^{(11)}$. Esto explicaría, en parte, que las tasas a nivel mundial son mayores en hombres que en mujeres en todos los rangos etarios y los resultados de este estudio no son la excepción. En el caso de los hombres, la tasa de mortalidad por suicidio para el año 2018 fue de 20,7 por 100.000 hombres, tasa casi 5 veces superior a la encontrada en mujeres (4,1 por 100.000 mujeres). Esta brecha entre hombres y mujeres ha sido relativamente uniforme durante el periodo con una leve disminución durante los últimos años, resultado similar a lo reportado por otros estudios ${ }^{(24)}$. Las diferencias en las tasas según sexo se mantienen de acuerdo a la evidencia en otras regiones del mundo, los hombres presentan gran parte de la proporción de todas las muertes por suicidio, con aproximadamente un $80 \%$ del total ${ }^{(3)}$.

Respecto de la edad, en los grupos etarios más jóvenes, se observaron la mayor cantidad de cambios en la tendencia (joinpoint) de las tasas de mortalidad específicas por edad según sexo. En el grupo de 15 a 19 años, se encontró un mayor cambio en el PCA, tanto en hombres como en mujeres, con disminuciones estadísticamente significativas para el último periodo (2007-2018), a diferencia de lo reportado en el estudio de Lee et al., en el que la tasa disminuyó solo en mujeres de edad similar ${ }^{(25)}$. Las tasas más altas, y que no presentaron cambios en la tendencia durante el periodo estudiado, se encontraron en hombres mayores de 60 años, siendo para el año 2018 casi 12 veces superior a la tasa en mujeres del mismo grupo de edad. El suicidio en adultos mayores posee diferentes implicancias respecto de los más jóvenes. El envejecimiento se caracteriza por la aparición de enfermedades crónicas, situaciones de dependencia, abandono, soledad y pérdida de vínculos sociales, lo que provoca malestar y dolor en individuos, estos serían factores desencadenantes en la decisión de suicidio $^{(26)}$. Además de lo señalado, esta diferencia entre tasas de suicidio de hombres y mujeres mayores de 60 años podría deberse a los métodos utilizados. La bibliografía reporta que las armas de fuego son el método más letal y el más comúnmente utilizado en hombres de este grupo ${ }^{(27,28)}$. Por otra parte, la bibliografía señala que el $61,6 \%$ de los adultos mayores que cometieron suicidio tenía al menos un diagnóstico psiquiátrico en comparación con adultos de edad mediana $(80,1 \%)^{(29)}$. Lo anterior podría indicar que existe una subrepresentación de enfermedades psiquiátricas en adultos mayores precisamente porque este tipo de padecimientos suele naturalizarse en pacientes de edad avanzada y, por tanto, muchas veces no se consulta al respecto.

El suicidio es un problema que posee múltiples factores causales ${ }^{(11,25)}$, por lo que resulta complejo explicar los cambios observados en la tendencia. Se ha identificado que uno de los factores altamente asociado a este problema es el deterioro en la salud mental. En Chile, actualmente no existe una política pública integral de salud mental; sin embargo, se han implementado diversas políticas que abordan el suicidio o la prevención de sus causas. Las políticas orientadas a salud mental implementadas en Chile han ampliado su oferta; sin embargo, esto no sería suficiente para lograr un efecto significativo en la disminución de las tasas de suicidio en hombres. Diversos estudios señalan que los hombres son más tardíos en manifestar síntomas depresivos y en consultar, lo que dificulta la pesquisa y tratamiento oportuno de trastornos mentales que puedan conducir al 
suicidio $^{(30)}$. Un estudio en Chile muestra que el $22,6 \%$ de los hombres reporta una percepción de bienestar mental o emocional menos que buena, y el 4,9\% declara que un médico le ha diagnosticado depresión ${ }^{(31)}$, este estudio también discute que la depresión entre los hombres podría estar subdiagnosticada y sin tratamiento debido a las diferencias de género en los síntomas y el afrontamiento. Además, el acceso a la atención en salud mental por parte de la población laboralmente activa y que pertenece al sistema público de salud, se encuentra obstaculizada por la limitada disponibilidad horaria que se ajuste a la escasa flexibilidad laboral. Esto afectaría más a hombres que a mujeres si se considera que son quienes constituyen la mayor fuerza laboral en Chile ${ }^{(32)}$, además en el tramo etario laboralmente activo ( 25 a 64 años) es en donde se presentaron las mayores alzas en las tasas de suicidio en hombres. Las crisis económicas, que contribuyen al desempleo y disminución del ingreso personal se han correlacionado con aumentos en el suicidio, particularmente en los hombres, aunque todavía no se ha establecido una relación causal directa ${ }^{(33,34)}$. Por lo tanto, es importante que la atención en salud tenga un enfoque de género, que considere las construcciones sociales que pueden influir en la salud mental y en la percepción de bienestar.

A pesar de que se observaron cambios en la tendencia de mortalidad por suicidio a nivel poblacional, el diseño del estudio no permite atribuir estos cambios a las intervenciones implementadas. La evidencia señala que las muertes por suicidio no se informan lo suficiente, especialmente en los países de ingresos bajos y medios ${ }^{(5)}$. En Chile, los registros de mortalidad presentan una alta calidad ${ }^{(17)}, \mathrm{y}$ se han incorporado mejoras en el registro y codificación de los certificados de defunción, disminuyendo los casos en los cuales se ignoraba la causa de muerte (accidental o intencional $^{(18)}$. No obstante, debemos señalar que durante los años 1997 y 2001 se codificaron eventos con intención indeterminada, en los cuales probablemente hubo lesiones autoinflingidas, que no fueron consideradas en el estudio. La interpretación respecto al aumento en la tasa de mortalidad durante el primer período debe ser cautelosa, ya que es posible que este aumento esté asociado al mejor registro y codificación de estos casos. La fortaleza del estudio contempla el estudio de la tasa de mortalidad por suicidio, autores reconocen que las investigaciones sobre suicidio realizadas en países de ingresos bajos y medios son limitadas ${ }^{(35)}$. Este estudio muestra los cambios en la tendencia de las tasas de mortalidad por suicidio, para un periodo de 21 años, según sexo y grupo de edad.

Actualmente existe consenso a nivel mundial en torno a cuáles debieran ser las medidas de prevención del suicidio a nivel de políticas públicas. Distintos organismos de salud han establecido criterios y sugerencias con relación a la investigación e intervención oportuna en este problema. Si bien Chile cuenta con programas de prevención del suicidio, es necesario evaluar la implementación y avanzar en estas medidas con un enfoque en las poblaciones identificadas con mayor riesgo de suicidio.

\section{REFERENCIAS BIBLIOGRÁFICAS}

1. World Health Organization. WHO Mental health action plan 2013-2020. Geneva; WHO; 2013.

2. Crosby AE, Ortega L, Melanson C. Self-directed violence surveillance: Uniform definitions and recommended data elements. Atlanta: Centers for Disease Control and Prevention, National Center for Injury Prevention and Control; 2011.
3. Värnik P. Suicide in the world. International Journal of Environmental Research and Public Health. 2012,9(3):760771. doi: 10.3390/ijerph9030760.

4. World Helath Organization. Preventing suicide: A global imperative. Washington DC: WHO; 2014.

5. Orpana HM, Marczak LB, Arora M, Abbasi N, Abdulkader RS, Abebe Z, et al. Global, regional, and national burden of suicide mortality 1990 to 2016: Sys- 
tematic analysis for the Global Burden of Disease Study 2016. BMJ. 2019;364:194. doi: 10.1136/bmj.194.

6. Bertolote JM, Fleischmann A. A global perspective in the epidemiology of suicide. Suicidologi. 2002;7(2):6-8. doi: 10.5617/suicidologi.2330.

7. Liu K. Suicide rates in the world: 1950-2004. Suicide \& Life-Threatening Behavior. 2009;39(2):204-213. doi: 10.1521/suli.2009.39.2.204.

8. Nahuelpan E, Varas J. El suicidio en Chile: Análisis del fenómeno desde los datos médico legales, Período 20002010, Actualización datos periodo 2011-2017. Santiago: Unidad de Estadística Servicio Médico Legal; 2018.

9. OECD. OECD Factbook 2014: Economic, Environmental and Social Statistics. Paris: OECD Publishing; 2014. doi: 10.1787/factbook-2014-en.

10. Nock MK, Borges G, Bromet EJ, Alonso J, Angermeyer M, Beautrais A, et al. Cross-national prevalence and risk factors for suicidal ideation, plans and attempts. British Journal of Psychiatry. 2008;192(2):98-105. doi: 10.1192/bjp.bp.107.040113.

11. Turecki G, Brent DA. Suicide and suicidal behaviour. The Lancet. 2016;387(10024):1227-1239. doi: 10.1016/ S0140-6736(15)00234-2.

12. World Health Organization. National suicide prevention strategies: progress, examples and indicators. Geneva: WHO; 2018.

13. Chile, Decreto 140 Modifica Decreto $N^{\circ} 70 \mathrm{DE}$ 2006, Reglamento de solariums o camas solares [Internet]. 2007 [citedo 10 sep 2019]. Disponible en: https://tinyurl.com/hrn4am74.

14. Chile, Ministerio de Salud. Programa Nacional de prevención del suicidio: orientaciones para su implementación. Santiago: Secretaría de Salud Pública; 2013.

15. Chile, Ministerio de Salud. Bases de datos Defunciones 1997-2017. Santiago: INE; 2019.

16. Organización Panamericana de la Salud. Clasificación CIE-10. Ginebra: OMS; 1992.

17. Núñez FML, Icaza NMG. Calidad de las estadísticas de mortalidad en Chile, 1997-2003. Revista Médica de Chile. 2006;134(9):1191-1196. doi: 10.4067/S003498872006000900016 .

18. Duarte Quapper DL. El suicidio en Chile: Estudio socio-demográfico y aproximación microsocial y psicológica a la violencia ejercida contra sí mismo. Valonia: Universidad Católica de Lovaina; 2007.

19. Instituto Nacional de Estadística. Proyecciones Demograficas y Vitales [Internet]. 2019 [citado 1 abr 2021]. Disponible en: https://tinyurl.com/5btwt7e2.

20. United Nations, Department of Economic and Social Affairs. World Population Prospects 2017 [Internet]. 2019 [citado 1 abr 2021]. Disponible en: https://tinyurl. com/u9dnk9vk.
21. Frome EL. The analysis of rates using Poisson regression models. Biometrics. 1983;39(3):665-674. doi: $10.2307 / 2531094$

22. Surveillance Research Program, National Cancer Institute. Joinpoint Regression Program Version 4.8.0.1 - April 2020; Statistical Methodology and Applications Branch [Internet]. 2020 [citado 1 abr 2021]. Disponible en: https://surveillance.cancer.gov/joinpoint/

23. Chile. Ley 17374 , Fija nuevo texto refundido, coordinado y actualizado del DFL. N ${ }^{\circ} 313$ de 1960, que aprobara la ley orgánica dirección estadística y censos y crea el instituto nacional de estadísticas [Internet]. 1970 [citado $10 \mathrm{abr}$ 2020]. Disponible en: https://tinyurl. com/5bczva6f.

24. Otzen T, Sanhueza A, Manterola C, Escamilla-Cejudo JA. Mortalidad por suicidio en Chile: Tendencias en los años 1998-2011. Revista Médica de Chile. 2014;142(3):305313. doi: 10.4067/S0034-98872014000300004.

25. LeeSU, ParkJI, LeeS, OhIH, ChoiJM, Oh CM. Changing trends in suicide rates in South Korea from 1993 to 2016: A descriptive study. BMJ Open. 2018;8(9):e023144. doi: 10.1136/bmjopen-2018-023144.

26. Jones AL, Pastor DK. Older adult suicides: What you should know and what you can do. Home Healthcare Now. 2020;38(3):124-130. doi: 10.1097/ $\mathrm{NHH} .0000000000000855$.

27. Substance Abuse and Mental Health Services Administration. Older Americans behavioral health, Issue Brief 4: Preventing suicide in older adults introduction and overview [Internet]. 2012 [citado 1 abr 2021]. Disponible en: https://tinyurl.com/2pacennu.

28. Curtin SC, Warner $M$, Hedegaard $H$. Increase in Suicide in the United States, 1999-2014. NCHS Data Brief. 2016;(241):1-8.

29. Kyung H-M, Park H-S, Bae S-M, Sung J-H, Kim I-B. Development of orthodontic micro-implants for intraoral anchorage. Journal of Clinical Orthodontics. 2003;37(6):321-328

30. Aravena JM, Gajardo J, Saguez R. Salud mental de hombres mayores en Chile: una realidad por priorizar. Revista Panamericana de Salud Pública. 2018;42:e121. doi: 10.26633/RPSP.2018.121.

31. Vidal C, Toro-Huerta C. Factores asociados al autorreporte del bienestar mental o emocional del hombre en Chile. Revista Panamericana de Salud Pública. 2018;42:e181. doi: 10.26633/RPSP.2018.181.

32. Roche AM, Pidd K, Fischer JA, Lee N, Scarfe A, Kostadinov V. Men, work, and mental health: A systematic review of depression in male-dominated industries and occupations. Safety and Health at Work. 2016;7(4):268283. doi: 10.1016/j.shaw.2016.04.005.

33. Borrell C, Marí-Dell'Olmo $M$, Gotsens $M$, Calvo $M$, Rodríguez-Sanz M, Bartoll X, et al. Socioeconomic inequalities in suicide mortality before and after the economic recession in Spain. BMC Public Health. 2017;17:772. doi: 10.1186/s12889-017-4777-7. 
34. Fountoulakis KN, Kawohl W, Theodorakis PN, Kerkhof AJFM, Navickas A, Höschl C, et al. Relationship of suicide rates to economic variables in Europe: 20002011. British Journal of Psychiatry. 2014;205(6):486496. doi: 10.1192/bjp.bp.114.147454.
35. Poorolajal J, Rostami M, Mahjub H, Esmailnasab N. Completed suicide and associated risk factors: A six-year population based survey. Archives of Iranian Medicine. 2015; 18(1):39-43.

\section{FORMA DE CITAR}

Vidal C, Faunes C, Toro Huerta C, Ruiz-Tagle C, Hoffmeister L. Cambios en la tendencia de mortalidad por suicidio en Chile, 1997-2018. Salud Colectiva. 2021;17:e3363. doi: 10.18294/sc.2021.3363.

Recibido: 22 dic 2020 | Versión final: 5 abr 2021 | Aprobado: 6 abr 2021 | Publicado en línea: 31 may 2021

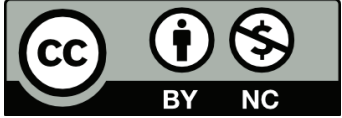

Esta obra está bajo una licencia de Creative Commons Reconocimiento-NoComercial 4.0 Internacional. Reconocimiento - Permite copiar, distribuir y comunicar públicamente la obra. A cambio, se debe reconocer y citar al autor original. No Comercial - Esta obra no puede ser utilizada con finalidades comerciales, a menos que se obtenga el permiso. 